dependence on imports for food and animal feeding stuffs from a world whose hungry multitudes (see (3) above) increase by 200,000 daily. How long can we rationally expect our own numbers to be maintained in this way, even at present levels? Surely they should rather (over a period of time) be related to the carrying capacity of our land.

It is important to distinguish this problem from that of overcrowding, which, though related, is a less acute one in survival terms. It may indeed be true that "many people . . . like living in crowded cities." They can do so only so long as food production from all known and hypothetical sources on this finite space-ship earth keeps the shelves of their supermarkets stocked. Reducing the number of regretted pregnancies is essential, but may not suffice. Truly informed choices as to their completed family size must be made by every parent in the land in the light of the facts above. These should be brought to them by every available means of publicity and education. "Doctors have unique opportunities to encourage young parents to think responsibly about the size of their families. Both the quality of life and the structure of society at the end of this century will depend on the answers found to population pressures in the next ten years" (B.M.F, 12 February, p. 391).

It is important therefore to find the right answers. Further discussions, about more than semantics, by the medical profession is nothing less than vital. I suggest that if the B.M.A. were to convene or support a symposium on this matter there would be widespread support for it and the potential to influence Government. Perhaps it might be timed in relation to a "population day," which is planned by the Conservation Society and related bodies for early May, 1973.-I am, etc.,

John GuIllebaud

Doctors and Overpopulation Group, Oxford 1 Meadows, D. H., Meadows, D. L., Randers, J.,

\section{Detecting Sickle Haemoglobin}

SIR,-Screening programmes for sickle haemoglobin should test for abnormal haemoglobins by methods that allow for the most accurate genetic diagnosis. Presently, the only such screening methods involve electrophoresis. It will be shown why primary sickle cell screening should not be performed by solubility tests, manual or automated. This thesis is contrary to that of a recent leading article (29 April, p. 246) and that of some other authors. ${ }^{1}$

The leading article suggested that special apparatus and experience are required for electrophoresis and also that the preparation of haemolysates and of buffers may be a disadvantage where technicians are lacking. Although the advantages of electrophoresis were recognized, the premise was offered that when cheapness, reliability, and the capacity for handling large numbers are the primary requirements the solubility test or some automated form may be preferred. Nevertheless, Autoanalyzers require technical competence and service, and the preparation of buffers for solubility tests and for electrophoresis requires comparable chemical knowledge.

The leading article also referred to a solubility test $^{2}$ that allegedly differentiates HbAS and HbSS genotypes, but it was not mentioned that this test does not discriminate $\mathrm{HbAS}, \mathrm{HbS} /$ thalassaemia, HbSC, or $\mathrm{HbS} /$ any abnormal haemoglobin.
Electrophoresis and solubility tests may be properly combined in sickle cell screening programmes by primary electrophoresis followed by testing samples that have abnormal haemoglobins with solubility test Thus, HbS, non-HbS sickle haemoglobins many of the more than 110 abnormal haemoglobins, and $\beta$ thalassaemia trait may be de tected. Samples with haemoglobins that migrate in the region of $\mathrm{HbS}$ and that are negative for sickle haemoglobin (and samples phenotypically HbSS) should be examined by acid agar electrophoresis or other tech niques for haemoglobins D, G, etc. Quantita tion of $\mathrm{HbA}_{2}$ and other haemoglobins and analyses for $\mathrm{HbF}$ are frequently indicated; other biochemical tests and pedigree analyses are often prerequisites for a final diagnosis. Genetic counseling is crucial in sickle cell screening programmes and is impossible without accurate genetic diagnoses. Solubility tests alone cannot provide this information.

A final overall dissent to the leading article is the implied licence for screening by per sonnel who have no technical skill. Programmes with such shortcomings are usually associated with similar deficiencies in sickle haemoglobin educational plans, genetic counseling, follow up, etc. The result has been psychological and socio-economic trauma through bogus education, erroneous diagnoses, and inept counseling. The victim of such programmes would have been far better off if they had never been tested. am, etc.,

\section{Department of Pathology,}

University of Chicago,

1 Nalbandian, R. M. Nichols, B. M., Heustis, A. E., Prothro, W. B., and Ludwig, F. E., fournal of the

2 Huntsman, R. G., Barclay, G. P. T., Canning, D. M., and Yawson, G. I., fournal of Clinical

\section{Dental Caries: An Alternative to Water Fluoridation}

SIR,-The World Health Assembly in 1969 recommended member states to introduce fluoridation of community water supplies where the total intake of fluoride was below the optimum level for protection against dental caries. A summary of the scientific basis for this recommendation is given in the monograph published by the W.H.O.1 and it is stated that this document is fully representative of informed scientific opinion throughout the world.

Fluoride ion is readily absorbed from the gastrointestinal tract. Any excess fluoride is speedily cleared from the blood, and readily excreted by the kidneys. In gross excess fluoride may give rise to skeletal fluorosis. The precise mechanism by which fluoride protects against caries is still unsettled.

In order, however, to be effective, and in order to carry public opinion with it, any such addition of fluoride should have regard to the following considerations: (1) it should be safe, and given in precise dosage; (2) it should be given only to those individuals who can benefit from it; (3) there should be no element of compulsion-those (or their parents) who do not desire fluoride should be able freely to decline participation in any such scheme; (4) any unused fluoride should not add to the pollution of the en- vironment; and (5) the relative costs of various vehicles for fluoride are of importance.

The compulsory fluoridation of the general drinking water does not conform with these conditions.

The effect of fluoride on the teeth of adults is minimal. Indeed some authorities would say it has no effect on adults, whose dental troubles are mostly periodontal. The question therefore arises whether some other vehicle might be used for fluoride. Milk has been considered as such a favourable vehicle. Like water it is a fluid, and when drunk as such there may also be a local action in the mouth cavity. It is also the food most universally used by pregnant women, infants, and children during the vital period of tooth formation and development. Milk is also a source of calcium and phosphorous which together with vitamin $D$ are required for the development of strong teeth and bones. In other words, milk is a major component of the diet at precisely the times in life when fluoride has its greatest effect-childhood, pregnancy, and the nursing period of the mother.

It was at one time thought that the addition of fluoride to milk might result in inadequate absorption of the fluoride because of the formation of insoluble calcium fluoride. The work of $\mathrm{Ziegler}^{2}$ makes it clear that this is not so and that absorption of fluoride when given in milk is good. If, theretore, fluoride can be given in carefully controlled doses to expectant mothers, infants, and children the fluoride will be given to the right recipients at the right time in the proper dosage. Parents who have a conscientious objection may withdraw their children from the scheme.

Projects of this kind have in fact been carried out successfully in the United States by Russoff and others, ${ }^{3}$ in Japan by Imamura, and in Switzerland by Ziegler." When milk has been used it has been in the same proportion of one part per million-the level at which it is placed, when the local authority approves it, in the public drinking water. In all these projects it has been shown conclusively that dental caries in children has been strikingly reduced.

It is suggested that the adoption of similar projects in this country, assisted by regular dental inspection at schools, would be a valuable measure and one which would fully meet the criticisms of those who object to fluoridation of the general water supply.I am, etc.,

CrrIL F. J. BARON

Esher, Surrey

1 Adler, P. et al., Fluorides and Human Health, Geneva, W.H.O. Monograph Series No. 59, Ziegler, E., Miteilungen der Naturwissenschaptlichen Gesellschaft in Winterthur, 1954, 28, 1 . Russoff, L. L., Konikof,, B. S., Frye, J. B., Johnston, J. 'E., and Frye, W.' W. American Imamura, Y., Fournal of Oral Disease Academy, Tokyo, 19539, 26, 1.

Ziegler, E., Bulletin der Schweizerischen Akademie

\section{Asian Immigrants}

SIR,-Can we be sure that adequate arrangements have been made for the medical examination of the Asians about to descend upon us? Provision must be made for accommodation of those undergoing tests, the results of which are not known immediately, 